\title{
Christine Scholz:
}

\section{Aus dem Jahresbericht der Informationsabteilung}

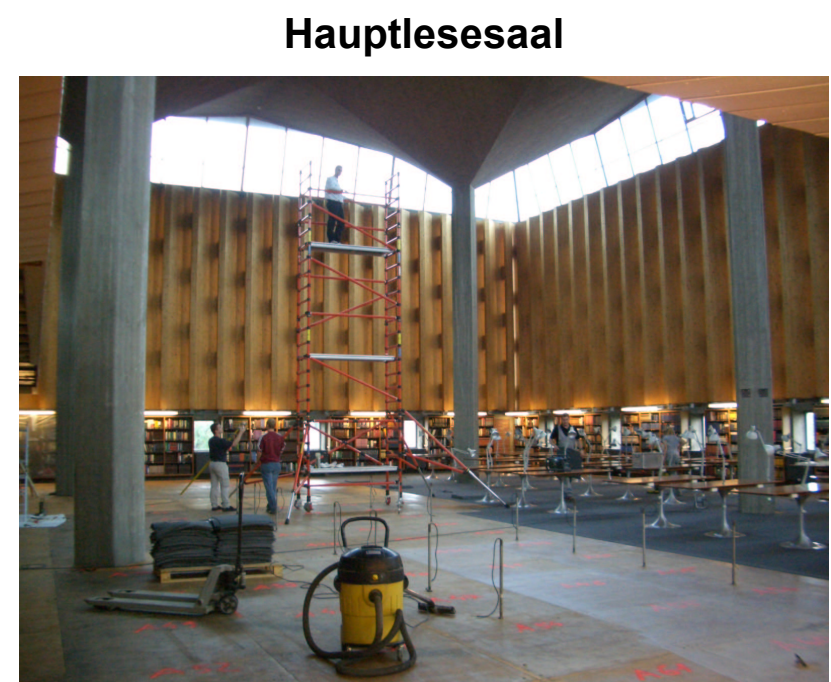

Abb. 1: Sanierung Hauptlesesaal

Prägend im vergangenen Jahr war die umfangreiche Sanierung des Hauptlesesaals.

Bereits im Januar 2004 begannen die abteilungsinternen Planungen. An diese Vorüberlegungen schlossen sich zahlreiche Gespräche mit Direktion, Verwaltung und dem Hochbauamt am. Am 24. Juli 2004 war es dann soweit: Der Hauptlesesaal wurde für eine 7wöchige Sanierung geschlossen, um die Asbestrückstände aus den Bodenplatten zu entfernen und neue Klimakanäle einzubauen. Gleichzeitig wurden mit dieser größeren Maßnahme die Brandschutzklappen ausgetauscht und an der Decke die Holzverkleidung erneuert. Im internen Mitarbeiterbereich konnten im Zuge der Sanierung die Buchrücklage mit Mitarbeiterplätzen vergrößert und der Schalter durch eine neue Theke sowie eine passende Schrankwand um den Aufzugsschacht optisch aufgewertet werden.

Die Sanierungsphase erforderte die komplette Räumung der unteren Lesesaalebene. Bis auf die Wandregale wurden alle Regale auf die freigewordene Fläche des Katalogbereichs verzogen. Für die Mitarbeiter des Lesesaals war dies mit umfangreichen Vorarbeiten verbunden, und der Umzug selbst musste beaufsichtigt werden.

Um unseren Benutzern während der Sanierungsphase die Präsenzbestände nutzbar zu machen, wurde die Möglichkeit vorab gegeben, Präsenzbestände nach Hause zu entleihen. Ausgenommen von dieser Regelung waren einige Sonderfälle (wie Loseblattsammlungen, Streckmappen) und die juristische Literatur, da diese von zahlreichen Benutzern gleichermaßen benötigt werden. Ingesamt wurden 460 Bände ausgeliehen, was von der Notwendigkeit einer Sonderausleihe zeugt. 


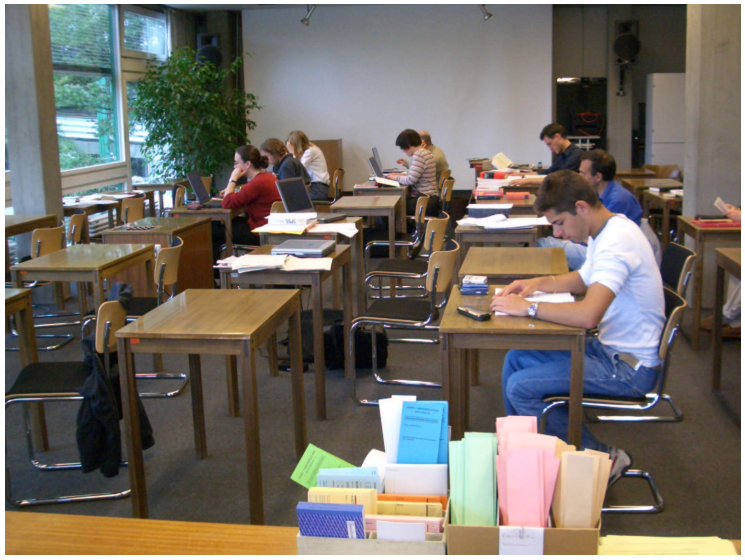

Abb. 2: Notlesesaal im Vortragsraum

In der Zeit vom 2. August bis 11. September 2004 wurde im Vortragsraum ein Notlesesaal eingerichtet, um den Lesesaalbetrieb in eingeschränktem Maß aufrechtzuerhalten. Dieser war montags bis freitags von 9-19 Uhr geöffnet und konnte auch an den beiden geöffneten Samstagen im September genutzt werden. Zwar waren die räumlichen Verhältnisse sehr beschränkt, jedoch konnten insgesamt 32 Benutzerarbeitsplätze eingerichtet werden, die nachmittags fast immer vollständig belegt waren. Im Notlesesaal wurden Fernleihbestellungen und Vormerkungen ausgegeben und Präsenzbestände zur Verfügung gestellt. EDV-Bestellungen mit Ausgabeort Lesesaal waren während dieser Zeit nicht möglich. In dringenden Fällen wurden jedoch Bücher aus dem Magazin geholt. Von den Präsenzbeständen wurden 813 Bände in den Notlesesaal zur Nutzung vor Ort entliehen.
Statistik

Der Präsenzbestand des Lesesaals umfasste zum Jahresende 45.360 Einheiten und damit 309 Einheiten mehr als im Vorjahr. Insgesamt wurden 4.705 Bände ein- bzw. ausgearbeitet. Erfreulich ist, dass auch in diesem Jahr die Verlustrate mit nur sechs vermissten Bänden sehr niedrig ausfiel. Die Buchsicherungsanlage im Eingangsbereich hat sich damit weiterhin bewährt.

An 251 Öffnungstagen wurden 103.398 Entleihungen gezählt, davon sind 90.138 per EDV aufgegeben worden. Bei den 13.260 Bestellungen mit Leihschein wurden 11.848 Mikroformen, 428 Sondersignaturen und 984 Zeitungen gezählt. Der starke Rückgang der Zeitungsbestellungen aus dem Magazin vor Ort ist vermutlich darauf zurückzuführen, dass im Sommer die Jahrgänge 1981-85 nach Fellbach ausgelagert wurden.

An schriftlichen Benutzerkontakten per Email und Post wurden 153 Anfragen bearbeitet. Hinzu kamen 370 Ausleihen und Kopienbestellungen im Rahmen der Dokumentlieferdienste. 


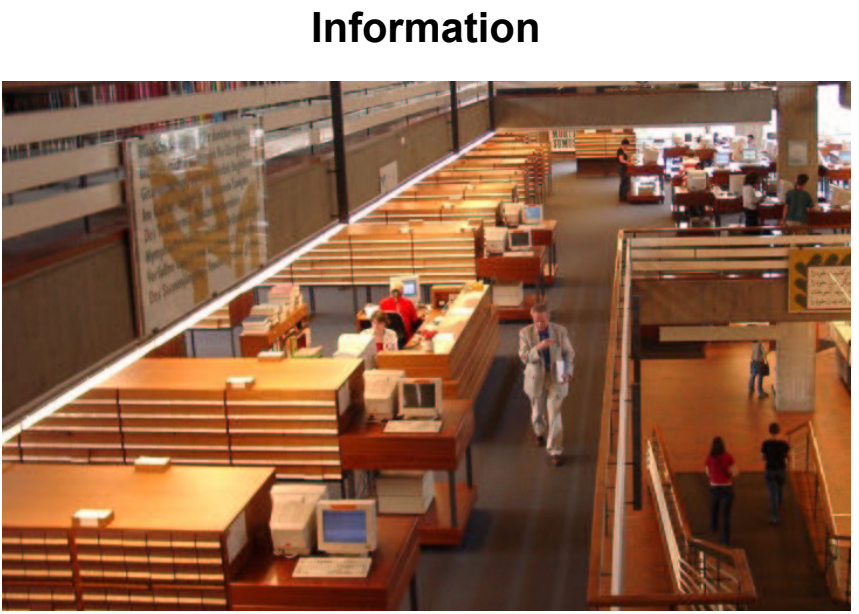

Abb. 3: Hauptebene mit Katalogen

Das vergangene Jahr war gekennzeichnet durch räumliche Umstrukturierungen auf der Hauptebene. Der Alphabetische Zettelkatalog wurde im ersten Halbjahr digitalisiert und damit abgebaut. Nach der Asbestsanierung konnte sich die Information im Herbst mit ihren Computerarbeitsplätzen in diesem Bereich einrichten, d.h. die Datenbankrechercheplätze der Empore wurden auf die Hauptebene verlegt. Diese Maßnahme hat sich sehr schnell als vorteilhaft für die Betreuung der Datenbankrecherchen erwiesen, da das Informationszentrum nun in unmittelbarer Nähe ist und somit ein direkter Benutzerkon- takt jederzeit möglich ist. Als weitere Verbesserung kam hinzu, dass die EDV-Abteilung den Benutzer-Desktop übersichtlicher und mit neuen Programmen und Zugriffsmöglichkeiten gestaltete, so dass die Arbeitsplätze nun einfacher zu bedienen sind und zusätzliche Ausgabemöglichkeiten (wie Brennen auf CD-ROM und Kopieren auf USB-Stick) genutzt werden können. Diese Maßnahmen führten insgesamt dazu, dass die Rechercheplätze und damit die Datenbanken nun wie angestrebt stärker von den Benutzern in Anspruch genommen werden.

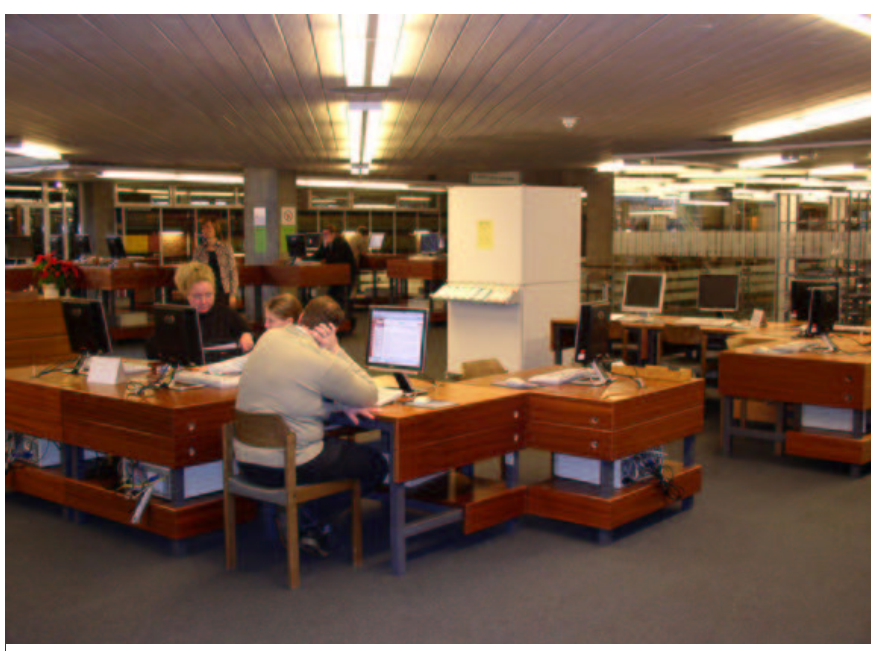

Abb. 4: Hauptebene mit Datenbankplätzen 
Mit dem Umzug der Datenbankplätze wurde die Besetzung der Informationstheke auf der Empore aufgegeben. Aus personellen Gründen war es bereits in den Vorjahren nicht möglich, zwei Informationstheken dauerhaft zu besetzen, so dass die Zusammenlegung beider Informationsstellen eine notwendige Konsequenz aus den bisherigen Erfahrungen war. Nun gibt es eine zentrale Anlaufstelle im Haus: das Informationszentrum.

\section{Statistik}

An Schulungen und Führungen für Multiplikatoren (Lehrer) wurden im vergangenen Jahr 41 Veranstaltungen angeboten. Neu hinzugekommen ist eine Intensivschulung, die jeden 5. Samstag angeboten wird. Diese Einführung in den Online-Katalog, DigiKat und Ausleihsystem richtet sich an Personen, die unter der Woche nicht an einem Abendtermin teilnehmen können. Die Resonanz war so positiv, dass diese Veranstaltung auch weiterhin angeboten werden soll.

Der Bestand der Nachschlagewerke ist in der Handbibliothek Information auf 25.249 Bände angewachsen und blieb damit weitgehend konstant. In der Bearbeitungsstatistik zeigt sich jedoch, dass insgesamt 742 Bände als Abgang und Zugang gezählt wurden.

Wie in den Vorjahren stieg die Zahl der eingehenden Email-Anfragen weiterhin an. Insgesamt wurden 503 Anfragen beantwortet; dies entspricht einer Zunahme von $17 \%$ gegenüber dem Vorjahr. Per Post dagegen wurden noch 68 Anfragen gestellt.

Auch bei den Kopieraufträgen ist eine starke Zunahme zu vermerken. Es wurden 193 Aufträge bearbeitet, was einen Zuwachs von 44\% gegenüber 2003 ausdrückt. An Benutzerwünschen gingen 636 Anschaffungsvorschläge ein, hier wiederum lag die Steigerung bei $23 \%$.

Für die Internetplätze wurden 2.374 neue Accounts von den Mitarbeitern angelegt oder bestehende Accounts verlängert. Mit 32.565 automatischen Freischaltungen an den Arbeitsplätzen ist dies ein gut genutzter Bereich der Bibliothek.

\section{Dokumentlieferdienste}

a) Fernleine

Seit dem Jahr 2003 beteiligt sich die WLB an der Online-Fernleihe des Bibliotheksservicezentrums Baden-Württemberg. Im vergangen Jahr konnte diese auf die bayerische Verbundregion ausgeweitet werden. Das bedeutet, dass nach negativem Durchlauf in der SWB-Region Bestellungen in den Bayerischen Bibliotheksverbund übergeben werden. Auch auf dem umgekehrten Weg erhalten wir nun Online-Bestellungen von bayerischen Bibliotheken.

Im vergangenen Jahr wurden im passiven Fernleihverkehr 6.529 Bestellun- 
gen über die Online-Fernleihe abgewickelt. In der aktiven Fernleihe waren es 21.567 Bestellungen. Aus dem ehe- maligen Projekt ist nun ein fester Bestandteil des Lieferspektrums an süddeutschen Bibliotheken geworden.

$\underline{\text { Statistik }}$

\begin{tabular}{|l|c|c|c|c|}
\hline & 2001 & 2002 & 2003 & 2004 \\
\hline Aktive Fernleine 1) & & & & \\
mit Verlängerungen & 47.366 & 48.338 & 42.234 & 52.450 \\
\hline ohne Verlängerungen & 37.146 & 37.721 & $\begin{array}{c}35.490 \\
\text { (online } \\
4.981)\end{array}$ & $\begin{array}{c}44.859 \\
\text { (online } \\
21.567)\end{array}$ \\
\hline Passive Fernleihe & 12.191 & 14.534 & $\begin{array}{c}13.505 \\
\text { (online } \\
427)\end{array}$ & $\begin{array}{c}19.216 \\
\text { (online } \\
6.529)\end{array}$ \\
\hline & & & $\begin{array}{c}23.205^{2)} \\
\text { z. T. manuell } \\
\text { gezählt }\end{array}$ \\
\hline
\end{tabular}

Tab. 1: Entwicklung der Bestellungen im Rahmen der Fernleihe 2001-2004

Sowohl in der aktiven als auch in der passiven Fernleihe sind starke Zuwächse zu verzeichnen. Über die aktive Fernleihe gingen 2004 im Vergleich zum Vorjahr 10.216 Bestellungen mehr ein. Dies entspricht einer Zunahme von $24 \%$. Bei den passiven Bestellungen gab es eine Zunahme um 5.711 Bestellungen, dies entspricht 42,5\%. b) Regionaler Leihverkehr

Über den Regionalen Leihverkehr (Landespost) werden ca. 200 Teilnehmerbibliotheken und 5 private Benutzer mit Literatur versorgt. Zu den Einrichtungen gehören überwiegend Stadtbüchereien, die $\mathrm{PH}$-Bibliotheken in Ludwigsburg und Schwäbisch Gmünd sowie einzelne Firmen.

\begin{tabular}{|l|c|c|c|c|}
\hline & \multicolumn{2}{|c|}{2003} & \multicolumn{2}{c|}{2004} \\
\hline Bestellungen & \multicolumn{2}{|c|}{30.134} & \multicolumn{2}{c|}{$14.779(49 \%)$} \\
\hline $\begin{array}{l}\text { positiv erledigt } \\
\text { (von WLB und anderen } \\
\text { Bibliotheken über WLB) }\end{array}$ & \multicolumn{2}{|c|}{$15.592(52 \%)$} & Köcher & Kopien \\
\hline & Bücher & Kopien & 13.616 & 1.163 \\
\hline
\end{tabular}

Tab. 2: Bestellungen und Lieferungen im Rahmen der Landespost 2003-2004

1) Gezählt wurden die konventionellen Bestellungen mit Fernleihschein und die Online-Bestellungen.

2) 2004 wurden erstmalig auch die nicht erledigten Bestellungen gezählt, die nicht von Bibdia erfasst wurden (+3.989 Bestellungen). 
Die Zahl der Bestellungen über die Landespost hat sich gegenüber dem Vorjahr leicht erhöht (+ 175 Bestellungen). Es konnten allerdings weniger Bücher und Aufsatzkopien (- 813) geliefert werden, da sehr viel mehr in den Bibliotheken ausgeliehen oder nicht am Standort vorhanden war.

\section{c) SUBITO}

Die Anzahl der eingehenden Bestellungen war 2004 gegenüber dem Vorjahr leicht rückläufig (- 327 Bestellungen). Dafür konnten jedoch mehr Bestellungen positiv erledigt werden (+ $561 \mathrm{Be}-$ stellungen), was zu höheren Einnahmen geführt hat. Positiv ausgewirkt hat sich, dass weniger Zeitschriften im Geschäftsgang waren. Der Anteil der entliehenen Bände hat sich dagegen erhöht.

\begin{tabular}{|l|l|l|}
\hline & 2003 & 2004 \\
\hline Bestellungen & 8.995 & 8.668 \\
\hline positiv erledigt & $\begin{array}{l}7.166 \\
(80 \%)\end{array}$ & $\begin{array}{l}7.727 \\
(82 \%)\end{array}$ \\
\hline pro Arbeitstag & 36,55 & 34,55 \\
\hline
\end{tabular}

Tab. 3: Bearbeitete Subito-Bestellungen 2003-2004 d) Bücherauto

Insgesamt wurden über die WLB im Jahr 2004 im Vergleich zum Vorjahr 4.267 Bände mehr transportiert. Bei der aktiven Fernleihe gingen dabei ca. $55 \%$ an Bibliotheken in BadenWürttemberg und $60 \%$ erhielt die WLB von Bibliotheken des Landes. Bei den Sendungen, die dem Bücherauto zunächst an das Transportzentrum der UB Karlsruhe mitgegeben werden und von dort per Container in andere Leihverkehrsregionen geschickt werden, ist eine Zunahme von 1.955 Bänden zu vermerken. Dies entspricht $24 \%$.

Zum 30. Juni 2004 musste die Bücherautolinie 2 (Saarland, Rheinland Pfalz, Mannheim, Heidelberg) eingestellt werden, nachdem sich die Bibliotheken in Rheinland Pfalz dem HBZ angeschlossen hatten und nun nicht mehr zu der Region des Südwestverbunds gehören. Die UB Mannheim und die UB Heidelberg sind seit 1. Juli 2004 mit Containern an das Transportzentrum in Karlsruhe angeschlossen, ebenso die saarländischen Bibliotheken. Damit können an diese Bibliotheken weiterhin Sendungen dem Bücherauto mitgegeben werden. Für die WLB hat sich geändert, dass sie die Abrechnung und Jahresstatistik des Bücherauto- und Containerdienstes und weitere Tätigkeiten (wie Fahrttermine festlegen, Bücherautofahnen erstellen) von der SLUB Saarbrücken übernommen hat. 


\begin{tabular}{|c|c|c|c|c|}
\hline & \multicolumn{2}{|c|}{2003} & \multicolumn{2}{|c|}{2004} \\
\hline \multirow[t]{2}{*}{ Versand mit Bücherauto } & \multicolumn{2}{|c|}{14.962} & \multicolumn{2}{|c|}{19.229} \\
\hline & $\begin{array}{l}\text { Aktive } \\
\text { Fernleihe }\end{array}$ & $\begin{array}{l}\text { Passive } \\
\text { Fernleihe }\end{array}$ & $\begin{array}{l}\text { Aktive } \\
\text { Fernleihe }\end{array}$ & $\begin{array}{l}\text { Passive } \\
\text { Fernleihe }\end{array}$ \\
\hline & 8.851 & 6.111 & 11.046 & 8.183 \\
\hline $\begin{array}{l}\text { Bibliotheken in BW und } \\
\text { Saarland }\end{array}$ & 5.617 & 3.130 & 6.123 & 4.936 \\
\hline $\begin{array}{l}\text { Weiterversand mit Con- } \\
\text { tainern } \\
\text { (Bayern, Sachsen, Nieder- } \\
\text { sachsen) }\end{array}$ & $\begin{array}{c}\text { Aktive } \\
\text { Fernleihe } \\
3.234\end{array}$ & $\begin{array}{c}\text { Passive } \\
\text { Fernleihe } \\
2.981\end{array}$ & $\begin{array}{c}\text { Aktive } \\
\text { Fernleihe } \\
4.923\end{array}$ & $\begin{array}{c}\text { Passive } \\
\text { Fernleihe } \\
\\
3.247\end{array}$ \\
\hline
\end{tabular}

Tab. 4: Versand an Bänden (ohne Kopien) über das Bücherauto BW mit Containerdiensten 2003-2004

\section{Birgit Mack:}

\section{Was ist neu auf der Homepage?}

Stand 5.9.2005

DBIS Datenbank-Infosystem

http://www.bibliothek.uni-regensburg.de/dbinfo/?bib_id=wlb

Seit August 2005 bietet die WLB Zugang zu DBIS, dem kooperativen DatenbankInfosystem. Eine Vielzahl der wissenschaftlichen Datenbanken ist frei im Internet nutzbar.

s. „Elektronische Angebote / Datenbanken“

\section{Sprechstunden der Fachreferenten/innen} http://www.wlb-stuttgart.de/kontakt/referate.htm

Die Fachreferenten/innen stehen für weitergehende und spezielle Fachauskünfte zu festen Servicezeiten oder nach Vereinbarung zur Verfügung.

s. „Kontakte / Fachreferate“ oder Faltblatt 19 Fachreferate - Sprechstunden

\section{Stellenangebote}

http://www.wlb-stuttgart.de/aktuelles/stellenangebot.html

Auf der WLB-Homepage gibt es nun auch eine dauerhaft eingefügte Seite „Stellenangebote“. Alle Arten von Stellenausschreibungen der WLB (für studentische Hilfskräfte, Auszubildende, Bibliothekare ...) werden hier veröffentlicht.

s. „Allgemeines / Stellenangebote“

\section{Kataloge und Bibliographien}

http://www.wlb-stuttgart.de/kataloge/opacs.html

Die frühere Seite „Online-Kataloge“ wurde übersichtlicher gestaltet und erhielt den neuen Namen „Kataloge und Bibliographien“.

Da die Anzahl der "Kataloge und Bibliographien der WLB" ständig anwächst, wurde dieser Abschnitt nochmals unterteilt in Hauptkataloge, Sonderkataloge, systematische Kataloge und Bibliographien.

Damit die Einträge schneller aufzufinden sind, gibt es ein Inhaltsverzeichnis am Seitenanfang.

s. „Elektronische Angebote / Kataloge und Bibliographien“ 